\title{
FEEDING DIFFICULTIES IN YOUNG PAEDIATRIC CARE SURVIVORS: A SCOPING REVIEW OF THE EVIDENCE
}

\section{Morton $\mathrm{K}^{1,5}$, Darlington $\mathrm{AS}^{2}$, Pappachan JV ${ }^{1,3}$, Marino $\mathrm{LV}^{2}, 4,5$}

\section{Background}

- The association between critical illness and feeding difficulties are well described in survivors of adult intensive care.

- $\quad$ Complex feeding disorders are described in children with congenital heart disease (CHD) and preterm infants, resulting in growth failure and significant parental anxiety.

There is a paucity of evidence describing the prevalence of feeding difficulties amongst paediatric intensive care (PICU) survivors and subsequent impact on family life.

Aims

- To complete a scoping review of evidence describing feeding difficulties amongst PICU survivors.

\section{Methods}

- Advanced literature search : six electronic databases plus grey literature and cross-referencing.

- Publications were eligible if they reported feeding difficulties and parental experiences amongst PICU and preterm graduates.

- Following initial title and abstract review, two reviewers independently screened full-texted publications and extracted study data.

\section{Results}

- All eight papers reported feeding difficulties in young survivors of intensive care, however the definitions and prevalence varied considerably.

- No paper described feeding difficulties in previously well babies/children.

- Papers described feeding difficulties in preterm infants and those with congenital diseases such as congenital heart disease.
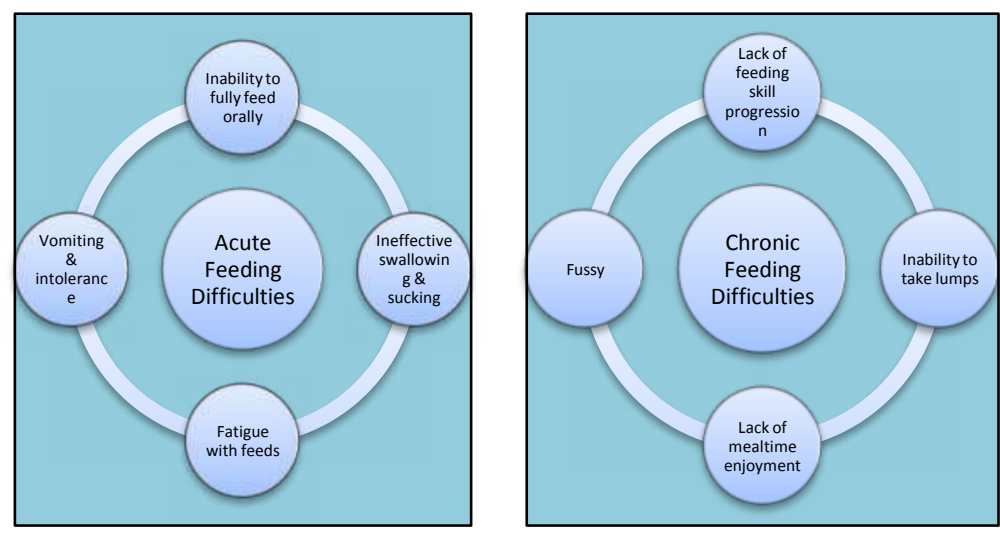

\section{Conclusions}

There is limited evidence describing feeding difficulties amongst young survivors of intensive care. As the majority of PICU admissions are infants, there is a need to explore this further in order to describe the prevalence of feeding difficulties and develop appropriate toolkits to reduce the burden of feeding difficulties post-discharge, reducing health care utilisation and parental anxiety.

PAPERS INCLUDED IN SCOPING REVIEW

Indramohan G, et al (2017) Identification of Risk Factors for Poor Feeding in Infants with Congenital Heart Disease and a Novel Approach to Improve Oral Feeding. Journal of pediatric nursing.35:149-54

Ross ES (2013) Feeding Outcomes in Preterm Infants After Discharge From the Neonatal Intensive Care Unit (NICU): A Systematic Review. Newborn and Infant Nursing Reviews.13(2):87-93.

Lutz KF (2012) Feeding Problems of NICU and PICU Graduates: Perceptions of Parents and Providers. Newborn and infant nursing reviews : NAINR.12(4):207-13.

Swift MC, Scholten I (2010). Not feeding, not coming home: parental experiences of infant feeding difficulties and family relationships in a neonatal unit. Journal of clinical nursing.19(1-2):249-58.

Jadcherla SR et al (2009) Feeding abilities in neonates with congenital heart disease: a retrospective study. Journal of Perinatology. 29. 112-118.

Kogon BE, et al (2007) Feeding difficulty in newborns following congenital heart surgery. Congenital heart disease. 2007;2(5):332-7.

Einarson KD, Arthur HM (2003). Predictors of oral feeding difficulty in cardiac surgical infants. Pediatric nursing. 29(4):315-9.

Hawdon et al (2000) Identification of neonates at risk of developing feeding problems in infancy. Developmenta medicine and child neurology.42(4):235-9.

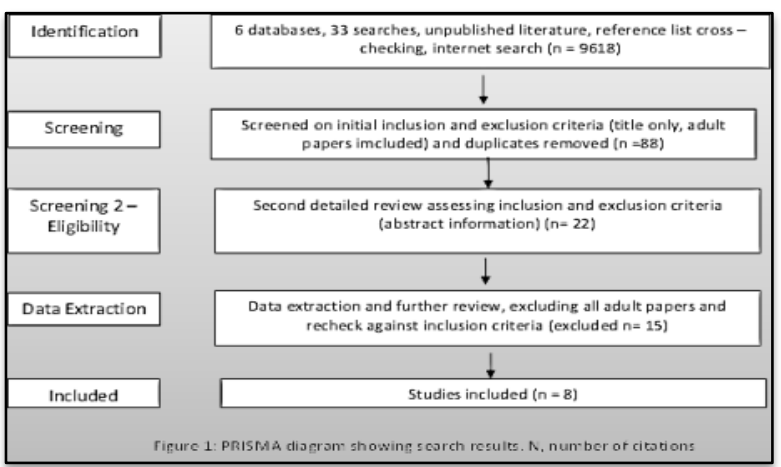

Figure 2: Overarching themes and interdependent relationships

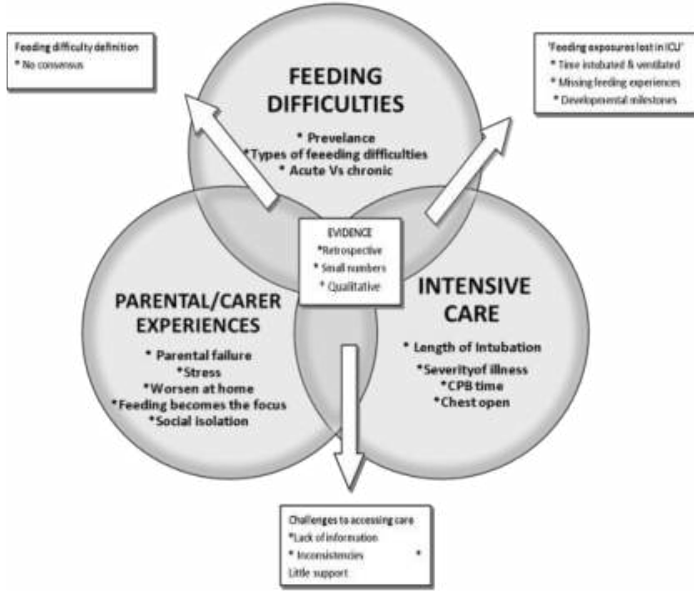

ey research priorities

Identifying \& defining feeding difficulties in young PICU survivors

Identifying risk factors
Effect of clinical care on feeding ability
Impact o

feeding difficulties

Affiliations: Paediatric Intensive Care Unit, University Hospital Southampton NHS Foundation Trust; ${ }^{1}$ Faculty of Health Sciences, University of Southampton ${ }^{2}$ Faculty of Medicine, University of Southampton ${ }^{3}$; Department of Dietetics/ SLT, University Hospital Southampton NHS Foundation Trust ${ }^{4}$; NIHR Biomedical Research Centre Southampton, University Hospital Southampton NHS Foundation Trust 